\title{
Status of iodine deficiency among pregnant mothers in Himachal Pradesh, India
}

\author{
Umesh Kapil ${ }^{1, *}$, Shyam Prakash ${ }^{2}$, Neha Sareen ${ }^{1}$, Ajeet Singh Bhadoria ${ }^{1}$, \\ Preetika Khenduja ${ }^{1}$, Sukirty Nigam ${ }^{1}$ and Jyoti Vijay ${ }^{1}$ \\ 'Department of Human Nutrition, All India Institute of Medical Sciences, Ansari Nagar, New Delhi - 110029, India: \\ ${ }^{2}$ Department of Gastroenterology and Human Nutrition, All India Institute of Medical Sciences, New Delhi, India
}

Submitted 24 June 2013: Final revision received 13 November 2013: Accepted 14 November 2013: First published online 6 March 2014

\begin{abstract}
Objective: Iodine is an essential micronutrient needed for the production of thyroid hormones. Pregnant mothers who are deficient in iodine provide less iodine to the fetal thyroid. This results in low production of thyroid hormones by the fetal thyroid, thereby leading to compromised mental and physical development of the fetus. The current study aimed to assess the current status of iodine nutrition among pregnant mothers in Himachal Pradesh, India, a known endemic region for iodine deficiency.

Design: Three districts, namely Kangra, Kullu and Solan, were selected.

Setting: In each district, thirty clusters (villages) were identified by utilizing the population-proportional-to-size cluster sampling methodology. In each cluster, seventeen pregnant mothers attending the antenatal clinics were included.

Subjects: A total of 1711 pregnant mothers (647 from Kangra, 551 from Kullu and 513 from Solan) were studied. Clinical examination of the thyroid of each pregnant mother was conducted. Spot urine samples were collected from ten pregnant mothers in each cluster. Similarly, salt samples were collected from eleven pregnant mothers in each cluster.

Results: Total goitre rate was $42 \cdot 2 \%$ (Kangra), $42 \cdot 0 \%$ (Kullu) and $19 \cdot 9 \%$ (Solan). The median urinary iodine concentration was $200 \mu \mathrm{g} / \mathrm{l}$ (Kangra), $149 \mu \mathrm{g} / \mathrm{l}$ (Kullu) and $130 \mu \mathrm{g} / \mathrm{l}$ (Solan). The percentage of pregnant mothers consuming adequately iodized salt (iodine content of $15 \mathrm{ppm}$ and more) was found to be $68.3 \%$ (Kangra), 60.3\% (Kullu) and $48 \cdot 5 \%$ (Solan).

Conclusion: Pregnant mothers in Kullu and Solan districts had iodine deficiency as indicated by a median urinary iodine concentration less than $150 \mu \mathrm{g} / \mathrm{l}$.
\end{abstract}

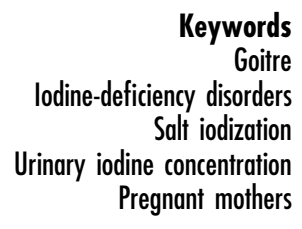

Iodine, an essential micronutrient, is required for the production of thyroid hormones and normal in utero neurological development. The dietary iodine requirement of pregnant mothers is higher $(250 \mu \mathrm{g} / \mathrm{d})$ than that of normal adults $(150 \mu \mathrm{g} / \mathrm{d})^{(1)}$. During pregnancy, the iodine requirement is increased by $50 \%$ due to (i) the physiological increase in maternal and fetal thyroid hormone production and (ii) the increase in renal iodine $\operatorname{loss}^{(2)}$. The thyroid hormones are crucial for brain and neurological development. The severe form of iodine deficiency leads to cretinism and mental retardation, which are irreversible. If the pregnant mother is iodine deficient, there is decreased synthesis of thyroxine by the fetal thyroid which leads to compromised mental and physical development of the fetus ${ }^{(3)}$.

Iodine deficiency affects 2 billion people worldwide and is the main cause of preventable mental impairment.
Every year, 38 million newborns in developing countries are affected by iodine deficiency ${ }^{(2)}$. In India, iodinedeficiency disorders (IDD) are a public health problem. Out of the 457 districts in the country, 344 districts have been surveyed for IDD, of which 263 districts have been found to be endemic for IDD ${ }^{(4)}$. The state of Himachal Pradesh is a known endemic region for iodine deficiency. There are studies available in the literature on the status of iodine nutrition among school-age children; however, there are limited data on the status of iodine nutrition among pregnant mothers from Himachal Pradesh.

The present study was conducted with the objective to assess the current status of iodine nutrition among pregnant mothers in three districts (Kangra, Kullu and Solan) of Himachal Pradesh so that the evidence could be provided to state health authorities of Himachal Pradesh to strengthen the IDD programme, if required. 


\section{Materials and methods}

\section{Study participants}

The study was undertaken in the year 2012 in Himachal Pradesh State. All three geographical regions, namely Kangra, Mandi and Shimla, were included. One district was selected from each region, i.e. Kangra (Kangra region), Kullu (Mandi region) and Solan (Shimla region). The study participants were selected by adopting a two-stage sampling technique. In each district, first thirty clusters (villages) were identified by utilizing population-proportional-to-size sampling methodology as recommended by WHO/UNICEF/International Council for the Control of Iodine Deficiency Disorders (ICCIDD) $)^{(1)}$. In the second stage, in each cluster (village), seventeen pregnant mothers who were attending the antenatal clinics were included. Pregnant mothers who were consuming drugs that could influence their thyroid status were excluded from the study.

\section{Clinical thyroid examination}

Clinical examination of the thyroid of each pregnant mother was conducted by two trained field investigators. The grading of goitre was done according to the criteria recommended jointly by WHO/UNICEF/ICCIDD: (i) grade 0 , not palpable and not visible; (ii) grade I, palpable but not visible; and (iii) grade II, palpable and visible $^{(1)}$. The sum of grades I and II provided the total goitre rate (TGR) in the study population. When in doubt, both investigators recorded the immediate lower grade. The intra- and inter-observer variation was minimized by repeated training of the two field investigators and by random examinations of goitre grades by the first author. The intra-observer variation was $10 \%$ and the inter-observer variation was $30 \%$ at the beginning of the study. This was minimized to less than $5 \%$ by repeated training before the data collection was started.

\section{Laboratory measurements}

In each cluster, a minimum of ten pregnant mothers were selected (from pregnant mothers who were enrolled for clinical thyroid examination) and casual urine samples were collected from them into plastic bottles with screw caps. Similarly, a minimum of eleven pregnant mothers were selected and salt samples were collected from them in auto-seal polythene pouches. Pregnant mothers were requested to bring four teaspoons of salt (about $20 \mathrm{~g}$ ) from their kitchen. The iodine content of the salt was analysed by the standard iodometric titration method ${ }^{(5)}$.

The urine samples were stored in a refrigerator until analysis. The analysis of urinary iodine concentration (UIC) was done within 2 months using the wet digestion method $^{(6)}$. Estimations of UIC and the iodine content of salt were done in the laboratory of the Human Nutrition Unit at the All India Institute of Medical Sciences.
Urine samples from Solan were found to have comparatively low UIC values in comparison with samples from Kangra and Kullu, and hence urine samples from Solan District were sent to the reference laboratory of the Indian Council of Medical Research, New Delhi, for re-analysis. In the current paper we present the UIC values obtained by the reference laboratory.

\section{Quality control measures}

The Internal Quality Control (IQC) methodology was adopted during UIC analysis. A pooled urine sample was prepared. This was considered the IQC sample, and it was stored in a refrigerator. It was analysed twenty-five times with standards and blank in duplicate. The mean UIC and standard deviation of this pooled sample were calculated. The $95 \%$ confidence interval for the mean UIC of the IQC sample was then calculated. This was used as the operating control range. The methodology adopted was as follows:

$$
\begin{aligned}
& \text { Sample mean }(X) \pm 2 \mathrm{SD} \\
& X-2 \mathrm{SD}=\text { the lower confidence limit or } \\
& \text { lower concentration value (LCV) } \\
& X+2 \mathrm{SD}=\text { the upper confidence limit or } \\
& \text { upper concentration value (UCV) }
\end{aligned}
$$

The operating control range for the IQC sample was between LCV and UCV. A regular linear graph paper was utilized to prepare Levey-Jennings plots. The mean UIC of the IQC sample was plotted as a continuous horizontal line on the $y$-axis. The LCV was plotted below the mean line on the $y$-axis scale and the UCV was plotted above the mean line on the $y$-axis scale. The $x$-axis was used to plot the date on which the IQC sample was analysed. This chart was used to plot the date-specific analysis. The pooled urine sample was analysed with every batch of samples submitted for UIC estimation. The UIC was obtained for the IQC sample analysed with each batch. If the UIC value of the IQC sample was between the two limit lines of LCV and UCV, then the UIC test was deemed in control and all results were accepted. If any value of the IQC sample was plotted outside the two limit lines of LCV and UCV, then the test was considered as out of control and the entire batch was repeated ${ }^{(7)}$.

\section{Sample size}

Keeping in view the anticipated prevalence of $5 \%$, confidence level of $90 \%$, absolute precision of $15 \%$ and design effect of 2 , a sample size of 500 was calculated to be required from each district from thirty clusters; i.e. a minimum of seventeen pregnant mothers from each cluster (510 pregnant mothers from each district). However, the present study included 647 pregnant mothers from Kangra, 551 from Kullu and 513 from Solan. 


\section{Results}

\section{Total goitre rate}

A total of 1711 pregnant mothers were included in the study for clinical examination of the thyroid gland from the districts of Kangra ( $n$ 647), Kullu ( $n$ 551) and Solan ( $n$ 513) in Himachal Pradesh. The mean age of the pregnant mothers was $25 \cdot 1$ (SD 3.3) years (Kangra), $23 \cdot 3$ (SD 3.4) years (Kullu) and $24 \cdot 7$ (SD 3.7) years (Solan).

The prevalence of goitre grade I was found to be 40.2\% (Kangra), $41 \%$ (Kullu) and 19.7\% (Solan). The prevalence of goitre grade II was found to be $2 \cdot 0 \%$ (Kangra), $1 \cdot 0 \%$ (Kullu) and $0 \cdot 2 \%$ (Solan). The TGR was found to be $42 \cdot 2 \%$ (Kangra), $42 \cdot 0 \%$ (Kullu) and $19 \cdot 9 \%$ (Solan) (Table 1).

\section{Urinary iodine concentration}

The UIC levels of the pregnant mothers and the percentage of pregnant mothers who consumed adequately iodized salt (salt with iodine content of 15 ppm or more) are depicted in Table 1. A total of 1118 urine samples were collected from the districts of Kangra ( $n$ 368), Kullu ( $n$ 439) and Solan ( $n$ 311). The median UIC levels were $200 \mu \mathrm{g} / \mathrm{l}$ (Kangra), $149 \mu \mathrm{g} / \mathrm{l}$ (Kullu) and $130 \mu \mathrm{g} / \mathrm{l}$ (Solan; Table 1).

The distribution of pregnant mothers according to trimester and the UIC level in each trimester are depicted in Table 2. In Kullu District, the median UIC value of pregnant mothers in the second and third trimester was 140.0 and $145 \cdot 0 \mu \mathrm{g} / \mathrm{l}$, respectively; while in Solan District, the median UIC value of pregnant mothers in the first, second and third trimester was $123 \cdot 7,135 \cdot 0$ and $132.5 \mu \mathrm{g} / \mathrm{l}$, respectively. These values are lower than $150 \mu \mathrm{g} / \mathrm{l}$, indicating iodine deficiency among pregnant mothers of Kullu and Solan districts.

\section{Discussion}

Pregnant mothers are the most vulnerable group for iodine deficiency as they have increased requirement for iodine due to the increase in BMR and increased renal excretion of iodine that accompany pregnancy. The assessment of UIC in pregnant mothers provides their current iodine nutritional status. It also suggests the likely occurrence of IDD among their newborns ${ }^{(1)}$. UIC is an indicator of recent dietary intake of iodine in the last $24 \mathrm{~h}$, as most of the iodine absorbed in the body appears in the urine. UIC is currently the most practical biochemical marker for iodine nutrition $^{(2)}$. According to the WHO, a median UIC level of $<150 \mu \mathrm{g} / 1$ among pregnant mothers indicates iodine deficiency in the community ${ }^{(1)}$. The present study found low iodine status among pregnant mothers in Kullu and Solan districts. This could be due to the consumption of salt with less than $15 \mathrm{ppm}$ iodine (the stipulated level in the country) by more than $30 \%$ of the pregnant mothers.

A recent study in the UK has documented that children of mothers who had iodine deficiency during pregnancy are more likely to have low verbal intelligent quotient and poor reading accuracy and comprehension ${ }^{(8)}$.

We are unable to compare the findings of our study on iodine status among pregnant mothers with other similar studies in India due to a lack of published data.

The TGR in a population indicates past iodine status and chronic low intake of iodine. Earlier studies conducted among pregnant mothers in the neighbouring states of Uttaranchal and Delhi reported a TGR of $15 \%$ and $1.9 \%$, respectively ${ }^{(9,10)}$.

Our earlier studies conducted among school-age children reported a TGR of $12 \cdot 1 \%$ (2000), 19.8\% (2007) and $15 \cdot 8 \%$ (2013) in Kangra District, $23 \cdot 4 \%$ (2013) in Kullu District, and 11.3\% (1999) and 15.4\% (2013) in Solan District ${ }^{(11-16)}$.

Table 1 Total goitre rate (TGR), median urinary iodine concentration (UIC) and percentage consuming adequately iodized salt (salt with iodine content of 15 ppm or more) among pregnant mothers in Kangra, Kullu and Solan districts of Himachal Pradesh, India, 2012

\begin{tabular}{|c|c|c|c|c|c|c|}
\hline & \multicolumn{2}{|c|}{ Kangra } & \multicolumn{2}{|c|}{ Kullu } & \multicolumn{2}{|c|}{ Solan } \\
\hline & $\%$ or median & $n$ & $\%$ or median & $n$ & $\%$ or median & $n$ \\
\hline TGR & $42 \cdot 2$ & 647 & $42 \cdot 0$ & 551 & $19 \cdot 9$ & 513 \\
\hline Median UIC $(\mu \mathrm{g} / \mathrm{l})$ & 200 & 368 & 149 & 439 & 130 & 311 \\
\hline Percentage consuming adequately iodized salt & $68 \cdot 3$ & 511 & $60 \cdot 3$ & 436 & $48 \cdot 5$ & 336 \\
\hline
\end{tabular}

Table 2 Distribution of pregnant mothers in different trimesters of pregnancy and median urinary iodine concentration (UIC) at each trimester in Kangra, Kullu and Solan districts of Himachal Pradesh, India, 2012

\begin{tabular}{|c|c|c|c|c|c|c|c|c|c|}
\hline \multirow[b]{2}{*}{ Trimester } & \multicolumn{3}{|c|}{ Kangra (n 368) } & \multicolumn{3}{|c|}{ Kullu (n 439) } & \multicolumn{3}{|c|}{ Solan ( $n$ 311) } \\
\hline & $n$ & $\%$ & Median UIC $(\mu \mathrm{g} / \mathrm{l})$ & $n$ & $\%$ & Median UIC $(\mu \mathrm{g} / \mathrm{l})$ & $n$ & $\%$ & Median UIC $(\mu \mathrm{g} / \mathrm{l})$ \\
\hline First & 19 & $5 \cdot 2$ & $200 \cdot 0$ & 46 & $10 \cdot 4$ & $162 \cdot 5$ & 35 & $11 \cdot 2$ & $123 \cdot 7$ \\
\hline Second & 219 & $59 \cdot \overline{5}$ & $200 \cdot 0$ & 263 & $60 \cdot 0$ & $140 \cdot 0$ & 186 & $59 \cdot \overline{8}$ & $135 \cdot 0$ \\
\hline Third & 130 & $35 \cdot 3$ & $200 \cdot 0$ & 130 & $29 \cdot 6$ & $145 \cdot 0$ & 90 & $29 \cdot 0$ & $132 \cdot 5$ \\
\hline
\end{tabular}




\section{Limitations of the study}

1. The intra- and inter-observer variation in goitre examination was controlled by repeated training and random examination of goitre grades by an expert. However, despite all of the training for quality control, there is still the possibility for misclassification of a normal thyroid gland as goitre grade I and vice versa.

2. We could not assess the size of the thyroid gland using ultrasound due to a lack of resources.

3. Iodine deficiency can be detected using maternal free thyroxine during the first trimester of pregnancy. We could not assess the same due to a lack of resources for this investigation.

\section{Conclusion}

The findings of the present study indicate that there is an urgent need to strengthen the IDD control programme for prevention of iodine deficiency among pregnant mothers in Kullu and Solan districts, Himachal Pradesh, as this could lead to IDD among their newborns. This can be achieved by efficient implementation of universal salt iodization with $15 \mathrm{ppm}$ iodine or more.

\section{Acknowledgements}

Sources of funding: The project was sanctioned by the Government of India, Ministry of Science and Technology, Department of Biotechnology (vide letter no. BT/PR-15435/ FNS/20/521/2011). The Government of India, Ministry of Science and Technology, Department of Biotechnology had no role in the design, analysis or writing of this article. Conflict of interest: The authors declare no conflict of interest. Ethics: The project was approved by the All India Institute of Medical Sciences, New Delhi. Authors' contributions: U.K.: study conception and design, preparation and finalization of the manuscript, overall scientific management, data analysis and interpretation. S.P.: study conception and design, data analysis and interpretation. N.S.: study conception and design, data collection, and preparation and finalization of manuscript. A.S.B.: study conception and design, data collection, and preparation and finalization of manuscript. P.K.: study conception and design, and data collection. S.N.: study conception and design, and data collection. J.V.: study conception and design, and data collection. Acknowledgements: The authors are grateful to the Government of India, Ministry of
Science and Technology, Department of Biotechnology for providing financial support.

\section{References}

1. World Health Organization/UNICEF/International Council for the Control of Iodine Deficiency Disorders (2007) Assessment of Iodine Deficiency Disorders and Monitoring Their Elimination. A Guide for Programme Managers. Geneva: WHO.

2. Li M \& Eastman CJ (2012) The changing epidemiology of iodine deficiency. Nat Rev Endocrinol 8, 434-440.

3. Hetzel BS (1989) The Story of Iodine Deficiency: An International Challenge in Nutrition. New Delhi: Oxford University Press.

4. Tiwari BK, Ray I \& Malhotra RL (2006) Policy Guidelines on National Iodine Deficiency Disorders Control ProgrammeNutrition and IDD Cell. New Delhi: Directorate of Health Services, Ministry of Health and Family Welfare, Government of India.

5. Karmarkar MG, Pandav CS \& Krishnamachari KAVR (1986) Principle and Procedure for Iodine Estimation - A Laboratory Manual. New Delhi: ICMR Press.

6. Dunn JT, Crutchfield HE, Gutekunst R et al. (1993) Methods for Measuring Iodine in Urine. A Joint Publication of WHO/ UNICEF/ICCIDD, pp. 18-23. Geneva: WHO.

7. Westgard JO, Barry PL \& Hunt MR (1981) A multi-rule Shewhart chart for quality control in clinical chemistry. Clin Chem 27, 493-501.

8. Bath SC, Steer CD, Golding J et al. (2013) Effect of inadequate iodine status in UK pregnant women on cognitive outcomes in their children: results from the Avon Longitudinal Study of Parents and Children (ALSPAC). Lancet 382, 331-337.

9. Pathak P, Singh P, Kapil U et al. (2003) Prevalence of iron, vitamin A, iodine deficiencies amongst adolescent pregnant mothers. Indian J Pediatr 70, 299-301.

10. Kapil U, Pathak P, Tandon M et al. (1999) Micronutrient deficiency disorder amongst pregnant women in three urban slum communities of Delhi. Indian Pediatr 36, 991-998.

11. Kapil U, Sohal KS, Sharma TD et al. (2000) Assessment of iodine deficiency disorders using the 30 clusters approach in district Kangra, Himachal Pradesh, India. J Trop Pediatr 46, 264-266.

12. Kapil U, Sharma TD \& Singh P (2007) Iodine status and goiter prevalence after 40 years of salt iodization in the Kangra district, India. Indian J Pediatr 74, 135-137.

13. Kapil U, Pandey RM, Kabra M et al. (2013) Status of iodine deficiency in district Kangra, Himachal Pradesh after 60 years of salt iodization. Eur J Clin Nutr 67, 827-828.

14. Chander S, Kapil U, Jain V et al. (2013) Iodine deficiency disorders in school age children in Kullu district, Himachal Pradesh. Indian Pediatr 50, 883-884.

15. Sohal KS, Sharma TD, Kapil U et al. (1999) Current status of prevalence of goiter and iodine content of salt consumed in district Solan, Himachal Pradesh. Indian Pediatr 36, 1253-1255.

16. Kapil U, Pandey RM, Jain V et al. (2013) Increase in iodine deficiency disorder due to inadequate sustainability of supply of iodized salt in District Solan, Himachal Pradesh. J Trop Pediatr 59, 514-515. 